1 Strain dynamics of specific contaminant bacteria modulate the performance of ethanol

\title{
2 biorefineries
}

4 Felipe Senne de Oliveira Lino ${ }^{\square 1}$, Maria-Anna Misiakou ${ }^{\square}$, Kang Kang ${ }^{\square 2}$, Simone S. Li ${ }^{\square 1}$, Bruno

5 Labate Vale da Costa $^{3}$, Thiago Olitta Basso ${ }^{4}$, Gianni Panagiotou ${ }^{2}$, Morten Otto Alexander

6 Sommer* ${ }^{1}$

7 Authors affiliations:

8 1: The Novo Nordisk Foundation Center for Biosustainability. Technical University of Denmark.

9220 Kemitorvet, Kongens Lyngby 2800. Denmark.

10 2: Leibniz Institute for Natural Product Research and Infection Biology. Beutenbergstraße 11A,

11 Jena 07745. Germany.

12 3: Escola de Engenharia de Alimentos da Universidade de Campinas. Rua Monteiro Lobato, 80.

13 13083-862 Campinas, SP. Brazil.

14 4: Departamento de Engenharia Química da Escola Politécnica da Universidade de São Paulo.

15 Universidade de São Paulo. Avenida Professor Lineu Prestes, 580. Cidade Universitária, 05508-

16 000, São Paulo, SP. Brazil.

17

$18 \square$ : These authors have contributed equally.

19 *: Corresponding author: Morten Otto Alexander Sommer - msom@bio.dtu.dk 


\section{Abstract}

23 Bioethanol is a viable alternative for fossil fuels, and its use has lowered $\mathrm{CO}_{2}$ emissions by over

24500 million tonnes in Brazil alone by replacing more than $40 \%$ of the national gasoline

25 consumption. However, contaminant bacteria reduce yields during fermentation. Our

26 understanding of these contaminants is limited to targeted studies, and the interplay of the

27 microbial community and its impact on fermentation efficiency remains poorly understood.

28 Comprehensive surveying and longitudinal analysis using shotgun metagenomics of two major

29 biorefineries over a production season revealed similar patterns in microbial community

30 structure and dynamics throughout the entire fermentation system. Strain resolution

31 metagenomics identified specific Lactobacillus fermentum strains as strongly associated with

32 poor industrial performance and laboratory-scale fermentations revealed yield reductions of up to

$334.63 \pm 1.35 \%$ depending on the specific contaminating strains. Selective removal of these strains

34 could reduce emissions from the bioethanol industry by more than $2 \times 10^{6}$ tonnes per year. Using

35 the large-scale Brazilian ethanol fermentations as a model system for studying microbiome-

36 phenotype relationships this study further demonstrates how high-resolution metagenomics can

37 identify culprits of large scale industrial biomanufacturing. 


\section{Introduction}

40 The Brazilian sugarcane ethanol production process generates more than 30 billion liters of

41 ethanol per year. This corresponds to more than $40 \%$ of all the energy consumed by light

42 vehicles in Brazil replacing the demand for almost 550 million barrels of oil per year ${ }^{1}$. The use of

43 this biofuel, either as a sole fuel or blended in the gasoline, results in reductions of more than

$4460 \%$ in total greenhouse gases emissions ${ }^{1,2}$. The sugarcane ethanol production process deploys

45 specific Saccharomyces cerevisiae strains, in very high cell density fed-batch fermentations

46 operated with cell recycling ${ }^{3,4}$. Usually the production season lasts for 250 consecutive days per

47 year, with an average mill performing up to 3 cycles of fermentation per day ${ }^{4}$. In total yeast cell

48 populations in a single fermentation exceed $10^{8}$ cells $\times \mathrm{ml}^{-1}{ }^{4}$. Still, contamination of this non-

49 sterile process remains a major problem leading to overall yield reductions of 3\%, corresponding

50 to over 960 million liters of ethanol ${ }^{5}$.

52 Contamination is mainly caused by lactic acid bacteria already present on the raw material which

53 tolerate ethanol, low $\mathrm{pH}$ and high temperature ${ }^{6}$. To control the bacterial contamination, yeast

54 cells are acid washed after every fermentation cycle before re-innoculation ${ }^{4}$. In spite of such

55 measures contamination continues to compromise the industrial process ${ }^{4,5,7}$. To further address

56 this issue, antibiotics and other antimicrobial compounds are used for contamination control.

57 However, antibiotic use raises serious concerns with regards to the global antibiotic resistance

58 crisis and also negatively impacts process economics. Given the continuous problems with

59 contamination there is an increasing need to understand the contaminating microbial community

60 in these bioprocesses as well as its effect on yeast fermentations ${ }^{3-5,8}$. 
62 Microbial communities are integral parts of most natural processes, from biogeochemical cycles

63 to the human health ${ }^{9,10}$, and the interactions among populations within these communities often

64 shape their functionalities and the surrounding environment ${ }^{11}$. To date, industrial fermentation

65 microbiomes have been studied with limited resolution applying either culture based methods ${ }^{12-}$

$66{ }^{16}$ or culture independent methods, like metabarcoding, focusing mainly in a specific process

67 steps ${ }^{17,18}$. These studies have either tried mainly to understand the overall composition of the

68 microbial community ${ }^{13,17,18}$, or to understand the impact of specific contaminant species in

69 ethanol fermentations at controlled laboratory environments ${ }^{6,19,20}$. Yet, new studies are needed to

70 correlate the composition of a complex community to actual industrial process performance, and

71 to discern the potential impact of strain-level variations in the functionalities of such

72 contaminating microbiomes ${ }^{21}$. Shotgun metagenomic sequencing could be a valuable tool for

73 pinpointing the contaminants that most significantly affect the performance of currently

74 established bioprocesses ${ }^{22}$.

76 In the present work, we have sampled all the unitary steps of the ethanol production process of

77 two mills in Brazil, during an entire fermentation season. We use shotgun metagenomics and

78 cultivation-based approaches to analyze the microbial community composition and pinpoint

79 specific detrimental strains configurations negatively impacting overall process performance, as

80 well as the mechanisms governing the community dynamics. This set of new information reveals

81 that higher-resolution metagenomics analysis are critical for understanding the dynamics of

82 microbial communities, and that strain level modifications are responsible for perturbing a

83 stablished microbiome. 


\section{Results}

\section{Independent sugarcane biorefineries share similar microbiome dynamics}

88 We selected two independently operated sugarcane ethanol mills in Brazil, hereafter referred to

89 as Mill A and Mill B, located over $300 \mathrm{~km}$ from each other but situated in similar climate regions

90 (Methods). The mills are also similar in overall production capacity and deploy the Melle-

91 Boinot fermentation process (Figure 1A $)^{4}$. In this process ethanol is produced via fast, high cell-

92 density, fed-batch fermentations. After the fermentation is finished yeast biomass is recovered

93 via centrifugation. This yeast cream is transferred to a separate vat, diluted with water and acid

94 washed to kill the contaminant bacteria. After this treatment, the yeast cream is pumped back to

95 the fermenters, and the process starts over for as many as 750 fermentation batches per year ${ }^{4}$.

96 The fermentation process is comprised of unidirectional steps providing defined sampling points

97 for our analysis (Figure 1A). To reduce potential bias introduced by seasonal variation, we

98 sampled each mill at three distinct timepoints throughout the production season (Supplementary

99 Table 1). We also collected fermentation metrics relevant to evaluate the ethanol production

100 process performance (Supplementary Table 1). In total, shotgun metagenomic sequencing was

101 applied to 56 samples yielding more than $2.8 \times 10^{5} \mathrm{Gbp}$ high quality data (Supplementary Table

102 2, Methods).

103

104 Focusing on the prokaryotic component of the metagenomes $(29.16 \pm 25.19 \%)$, we found

105 Firmicutes to be the most prevalent phylum, owing mostly to a high abundance of

106 Lactobacillaceae species (Figure 1B, Supplementary Table 3, Supplementary Table

$1074)^{6,13,18,19}$. Microbial communities from fermentation broth were found to be the most diverse and

108 least similar to those in the rest of the fermentation process (Supplementary Figure 1). This 
109 difference is mainly driven by the overall dominance of lactobacilli during the fermentation

110 process, since these organisms are best fit to handle the low oxygen and $\mathrm{pH}$, and high

111 temperature and ethanol concentrations, found in industrial fermentations ${ }^{19}$. In addition, we

112 observed that the composition of the contaminant microbiomes across all industrial process steps

113 were highly similar by the end of the production season (final sampling timepoint). Inspection of

114 the collected data revealed that both mills were operating below maximum capacity due to lack

115 of raw material, which resulted in microbial biomass being left idle for longer periods in the

116 fermentation vessels. Irrespective of this the microbial communities of both mills were not found

117 to differ significantly when comparing across process steps and timepoints ( $p=0.293$,

118 PERMANOVA; Supplementary Figure 2).

120 The majority of the microbial communities, throughout the entire fermentation process, were

121 found to be dominated by either L. fermentum or L. amylovorus (Figure 1B). These two species

122 have independently been described as contaminants in other ethanol fermentation processes ${ }^{6,23,24}$.

123 We find that these two species constitute more than $50 \%$ of the relative abundance of these

124 contaminating communities, demonstrating how uneven such communities are. Interestingly,

125 when comparing relative abundances, we detected an inverse relationship between the two

126 species during the fermentation stages, which suggests competition between L. fermentum and $L$.

127 amylovorus during the ethanol production process (Spearman's correlation $\rho=-0.908$; FDR <

$1284.3 \times 10^{-14}$, Figure 1C). For both mills, a similar pattern was observed, where L. amylovorus

129 dominates at the beginning of the fermentation followed by a decline in its relative abundance in

130 the community throughout the fermentation process. 
132 In contrast L. fermentum expands its relative abundance in the community from the beginning to

133 the end of the fermentation. The tolerance of L. fermentum towards high ethanol titres ${ }^{13}$ might

134 partly explain its higher relative abundance in the community during the final stages of

135 fermentation. The heterofermentative metabolism of L. fermentum might also be better suited to

136 compete with yeast for nutrients in this fermentation setup, compared to the homofermentative

137 metabolism of L. amylovorus ${ }^{6}$.

A

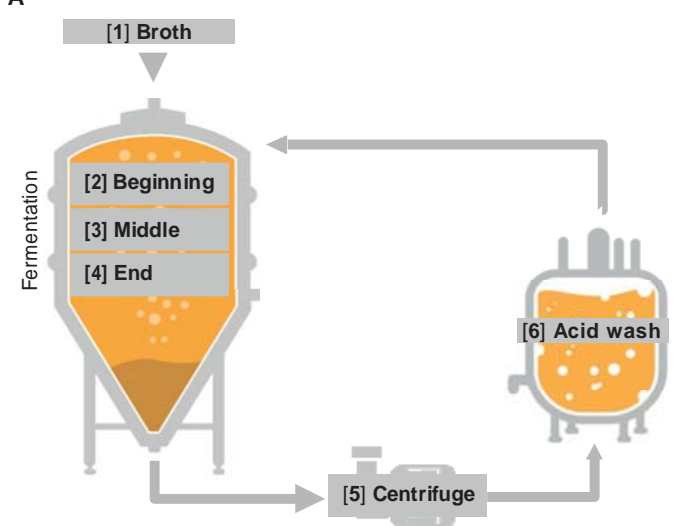

C

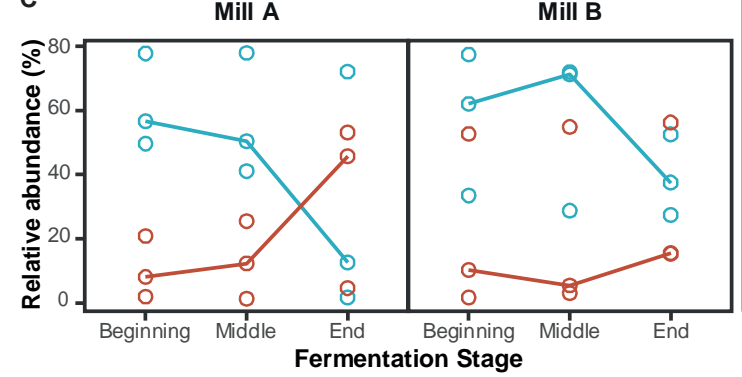

B

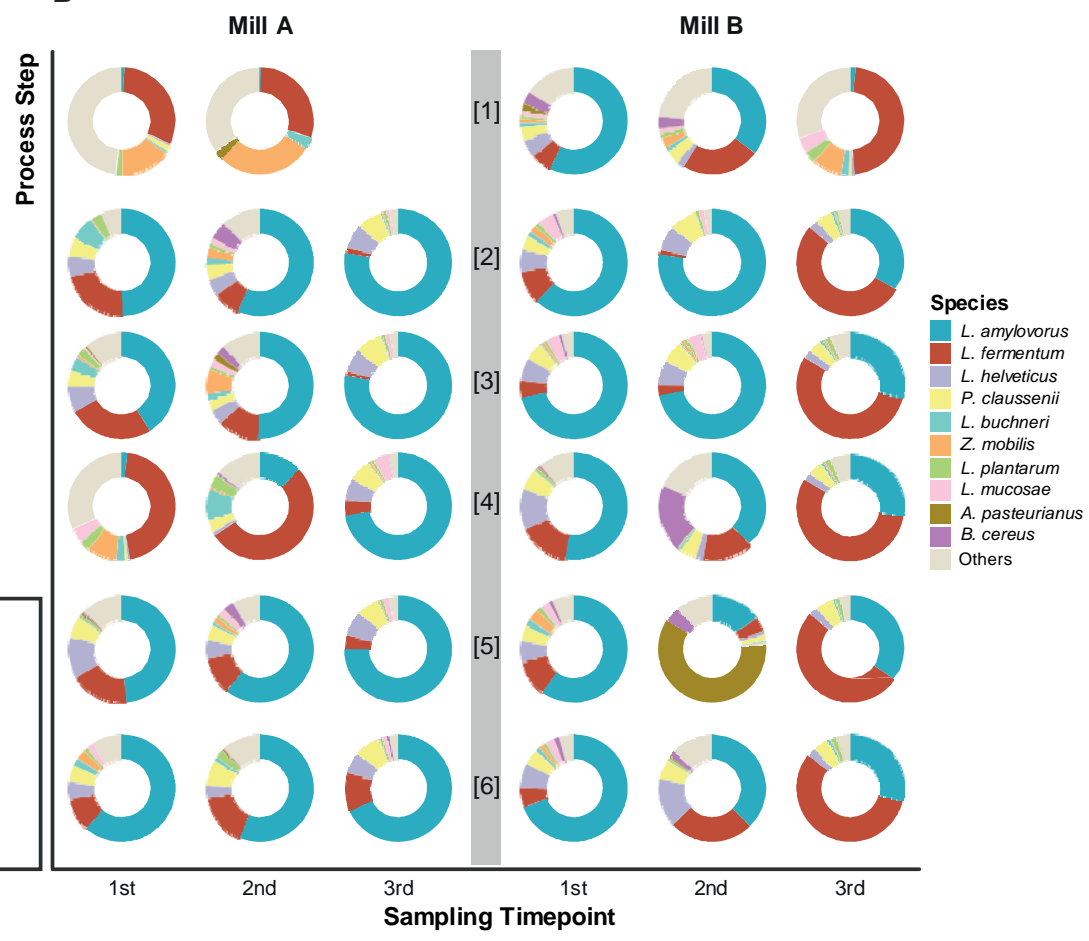

138

Figure 1: Sampling strategy and competition between $L$. amylovorus and $L$. fermentum in

the bioethanol fermentation process. A: Schematic of the fermentation process, indicating sampled steps: 1. Broth: Feeding line with fresh fermentation media; 2. Fermentation beginning: the beginning until middle of vessel feeding time (e.g. time 0 to $1.5 \mathrm{~h}$ of feeding); 3 . Middle: the middle until the end of feeding (e.g. time 1.5 to $3 \mathrm{~h}$ of feeding); 4: End: the final hours of fermentation, after feeding ceased; 5. Centrifuge: the yeast cream, resulting from the separation 
146 of the wine sent to distillation; 6. Acid wash: samples collected by the end of this treatment.

147 Vector images were obtained from Flaticon (www.flaticon.com). B: Species-level microbial

148 community showing the 10 most prevalent contaminants across sampling timepoints (x-axis) and

149 process steps (y-axis) in Mills A (left) and B (right), expressed as relative abundances. Beige

150 color indicates all remaining species of the community. C: Relative abundances of $L$.

151 amylovorus (blue) and L. fermentum (red) across the fermentation steps, as shown in A.

152 Spearman's correlation analysis of their relative abundances suggests competition among these

153 two species $\left(\mathrm{r}=-0.908 ;\right.$ FDR $\left.<4.3 \times 10^{-14}\right)$. Due to low biomass, DNA extraction was not

154 possible for sample [1] for the $3^{\text {rd }}$ sampling timepoint from Mill A. During the $3^{\text {rd }}$ sampling

155 timepoint, Mill B was operating below its maximum capacity. Biomass was left idle for longer

156 periods in the vessels. This might explain why all the process steps are similar in community

157 composition.

159 Microbial community composition affects industrial process performance

160 To establish if the dynamics observed within the contaminant microbial community were

161 associated with environmental factors or overall fermentation yield, we incorporated

162 fermentation data and industrial performance indicators into our analyses (Figure 2A,

163 Supplementary Table 1). Throughout the bioethanol production season, increased ethanol yield

164 was found to strongly associate with lower acidity titres in the fermentation (Spearman's

165 correlation $\rho=-0.84$, FDR $=2.09 \times 10^{-5}$ ), while increases in bacteria negatively impacted the

166 yeast viability $\left(\rho=-0.72, \mathrm{FDR}=2 \times 10^{-3}\right)$. 
Based on these findings, we then sought to identify contaminant species that were associated with fermentation performance (Methods). We did not expect to find strong associations between the species abundance and ethanol yield, due to well-documented caveats of ethanol

171 quantification methods ${ }^{25}$. Yet, L. fermentum was found to be strongly associated with increased 172 acidity titres, which hamper ethanol yield (Figure $\mathbf{2 A}$ and 2B, Spearman's correlation $\rho=$ 1730.72 , FDR $<1.50 \times 10^{-6}$ ). We also identified a number of bacterial species that were not 174 previously associated with impacts over industrial ethanol production, like Geobacillus 175 stearothermophillus. This species is a thermophile capable of producing a vast array of 176 cellulolytic enzymes ${ }^{26}$. Its presence in the fermentation suggests that this industrial environment 177 is a potential untapped source for novel industrially relevant enzymes.
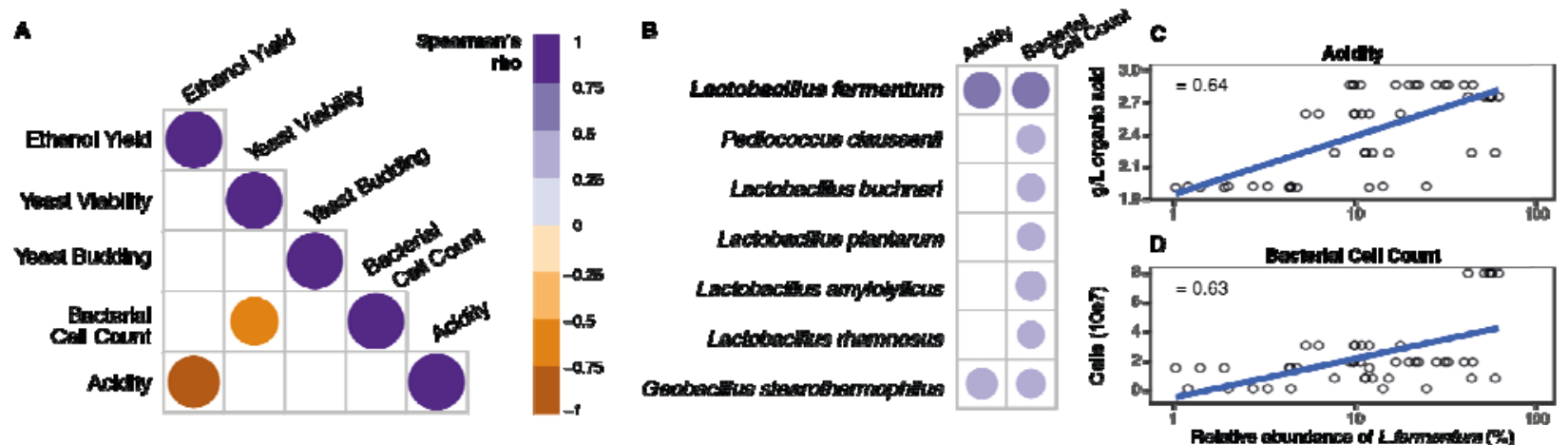

Figure 2: Microbial species and other factors that influence industrial fermentation performance. A: Fermentation parameters that showed strong associations throughout the

181 production season. Positive correlations are depicted as purple, whereas negative correlations are

182 depicted as orange. Size of each point denotes the strength of correlation. Increased acidity is

183 linked with lower ethanol yield, and increased number of bacterial cells reduces yeast viability.

184 B: Microbial species associated with fermentation performance. Increased L. fermentum during

185 fermentation in strongly linked to higher acidity titres. Species are ordered by decreasing relative

186 abundance. C: Correlation between relative abundance of $L$. fermentum and acidity ( $\rho=0.64$; 
187 FDR $\left.=1.5 \times 10^{-5}\right)$. D: Correlation between relative abundance of L. fermentum and bacterial cell

188 count $\left(\rho=0.63 ; \operatorname{FDR}=9.5 \times 10^{-6}\right)$.

190 The fate of fermentations is defined by the interplay of different $\mathrm{L}$. fermentum strains 191 during the acid wash

192 Given its strong association with increased acidity, which creates detrimental fermentation 193 conditions $^{19}$, we sought to establish if the negative effect conferred by L. fermentum was 194 mediated by strain variants of this bacteria. SNV profiling of core L. fermentum genes revealed 195 the presence of 3 distinct strain families within the species (Methods, Supplementary Figure 3, 196 Supplementary Table 5). Applying these profiles to our samples enabled insights into the strain 197 population structure and dynamics during industrial fermentation and its impact on performance.

199 To more accurately evaluate and compare the performance amongst different fermentation 200 batches $^{25}$, we devised a composite parameter that incorporated ethanol yield measurements, as 201 well as parameters related to the biological catalyst quality (yeast viability) and potential hazards 202 due to microbial contamination (bacterial cell counts, fermented broth acidity). We termed this 203 metric the Industrial Performance (Methods).

205 The correlation of strain clusters with different components of industrial performance (i.e. acidity 206 and yeast viability) suggests that specific phenotypes are more detrimental to the industrial 207 process. Organic acids are the main metabolites produced by L. fermentum, and is involved in the 208 reduction of yeast viability and metabolic capacity due to intracellular acidification and anion 209 accumulation $^{27}$. Acidity is, therefore, an indirect measurement of its metabolism, and its negative 
210 impact on ethanol yield and industrial performance demonstrates that the metabolism and growth

211 of bacteria is critical for this industry.

213 For both mills, a similar trend was observed in which poorer industrial performances are

214 observed when strain clusters 1 and 3 are dominant in the L. fermentum population (>50\% in

215 relative abundance). In contrast, dominance of L. fermentum strain cluster 2 is linked with

216 improved industrial performance ( $\mathrm{p}<0.02$ in both mills; Wilcoxon rank-sum test, Figure 3A).

217 This new evidence suggests that, contrary to what is currently considered in the literature, the

218 impact of L. fermentum in the fermentations is driven by its population's strain composition,

219 rather than its abundance in the process ${ }^{19,28-32}$. It also suggests that current contamination control

220 practices need to take into account the strain dominance, in order to choose the best molecule or

221 mode of application. This can only be achieved with higher resolution diagnostics.

222 To understand the mechanism underlying these different population composition in fermentation

223 with high and low industrial performance we analysed the L. fermentum population composition

224 for each unitary step of the fermentation process. The population composition during the

225 beginning of the fermentation is crucial for defining the industrial performance of the

226 fermentation batch. More specifically, the relative abundance of strain cluster 2 is directly

227 correlated with higher industrial performances scores $(\rho=0.73$, FDR $=0.003)$, and the

228 abundance of strain clusters 1 and 3 are linked to lower industrial performance scores $(\rho=-0.51$,

229 FDR $=0.042 ; \rho=-0.70$, FDR=0.006, respectively) (Supplementary Table 6).

231 Notably, the strain level composition of strain clusters 1 and 2 in the acid wash tank, the unitary 232 step immediately prior to the new fermentation batch, is directly correlated with their relative 
233 abundance in the beginning of the fermentation defining the strain composition of new batches $(\rho$

$234=0.60$ and FDR $=0.016$ for cluster $1 ; \rho=0.85$ and FDR $=0.0002$ for cluster 2$)($ Supplementary

235 Table 6). The direct correlation between acid wash and the beginning of the fermentation

236 suggests that the microbial community composition is mainly driven by cell recycle, rather than

237 the addition of novel contaminants through the broth. This hypothesis is further backed by the

238 higher dissimilarity observed in the broth, when compared to the other fermentation steps

239 (Supplementary Table 1), suggesting a more diverse and different community composition than

240 the one found in actual fermentations.

242 The population dynamics of the two strain clusters suggest direct competition in the acid wash $(\rho$

$243=-0.81$, FDR $=1.6 \times 10^{-4} ;$ Figure 3B $)$. The outcome of this competition between closely related

244 strain clusters in the acid wash is decisive for the industrial performance of the following

245 fermentations.

247 Focusing on identifying potential process parameters that could influence this strain level

248 dynamics we have analysed the correlation between the strain level compositions of $L$.

249 fermentum populations against all registered metadata. We have also broken down this analysis

250 into the specific operational processes, in order to identify any trends correlated with a specific

251 step of the ethanol production process. The fermentation temperature found in individual vessels

252 is the key driving factor defining L. fermentum strain level composition in the acid wash. More

253 specifically, higher temperatures throughout the fermentation lead to a higher relative abundance

254 of strain cluster 1 in the acid wash, after the fermentation $(\rho=0.55$, FDR $=0.0001$; Figure 3C),

255 favouring this specific cluster. In that way, the different fermentation batches are intimately 
256 connected, and the impact on process is more dependent on the established microbiome rather

257 than novel contaminants entering via fermentation broth.

A

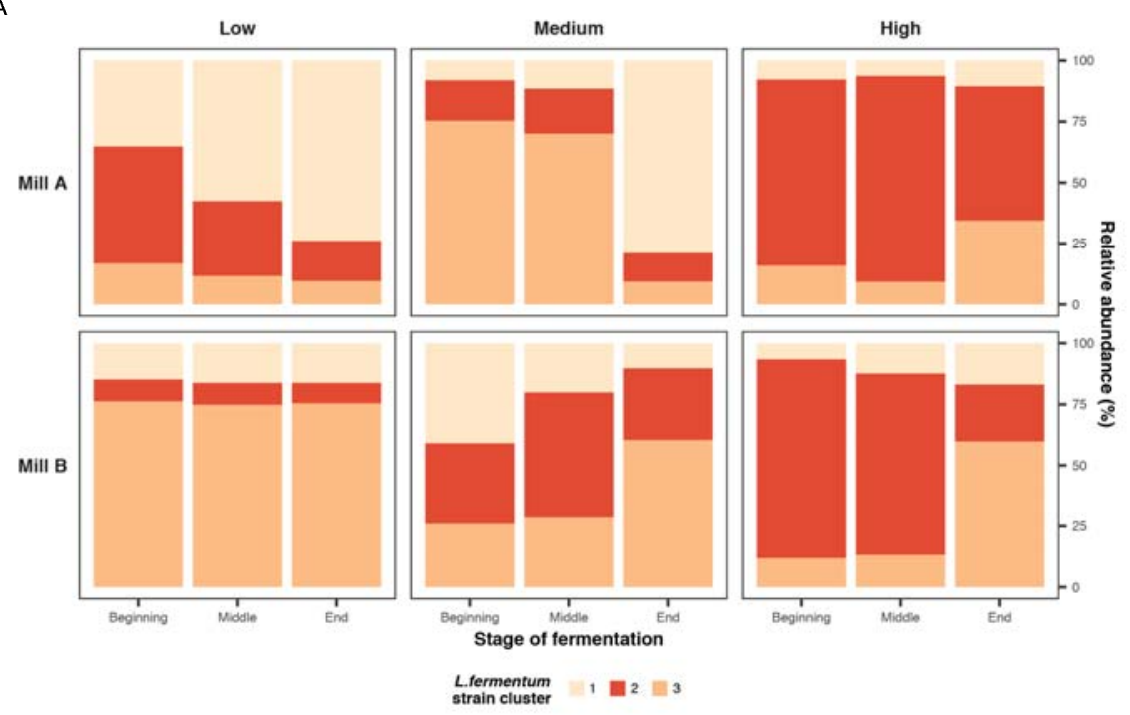

B

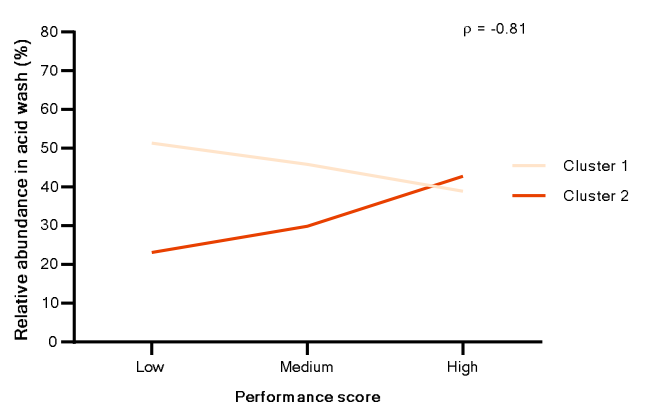

C

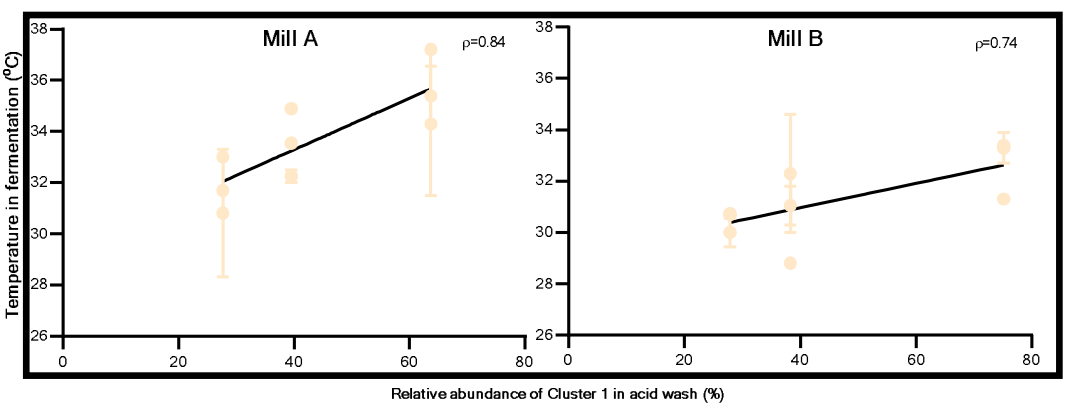

Figure 3: L. fermentum strain dominance is associated with process performance. A: The

262 fermentation steps (beginning, middle and end) against industrial performance score (Low,

263 Medium and High). Strain cluster 2 dominates high-performance batches during most of the

264 fermentation process $(\mathrm{p}<0.02)$, whereas clusters 1 and 3 reach their lowest relative abundances in

265 these high-performance batches. B: The interplay between L. fermentum strain clusters 1 and 2 in

266 the acid wash are intimately connected the population composition in the beginning of the 
267 fermentation $(\rho=0.52$ and FDR $=0.021, \rho=0.82$ and FDR $=0.004$ for clusters 1 and 2 ,

268 respectively) and with performance scores. The competition between these two strain clusters

269 decides the fate of the next fermentation batch, being mutually exclusive in the acid wash $(\rho=-$

2700.81, FDR $\left.=1.6 \times 10^{-4}\right) . \mathbf{C}$ : Higher fermentation temperatures privilege the detrimental strain

271 cluster 1, being directly correlated with its higher relative abundance in the acid wash, and in

272 subsequent fermentations, for both mills $(\rho=0.84$, FDR $=0.0048$ for mill $A ; \rho=0.74$, FDR=

2730.0185 for mill B).

274

275 Temperatures above $32^{\circ} \mathrm{C}$ during the fermentation process are correlated with higher relative

276 abundances of cluster 1, and with lower performance scores. Keeping the fermentation below

277 this temperature threshold would, therefore, favour strain cluster 2, a less detrimental cluster of

278 L. fermentum.

280 To test if our hypothesis is correct we sought to investigate if there are strain level variance in the

281 temperature dependence of the growth rate of different Lactobacillus strains. In laboratory

282 conditions it was also possible to replicate this phenomenon. The growth rate $\left(\mathrm{h}^{-1}\right)$ of actual

283 industrial lactobacilli isolates was compared in two different temperatures $\left(30^{\circ} \mathrm{C}\right.$ and $\left.37^{\circ} \mathrm{C}\right)$.

284 While L. amylovorus shows a growth rate $14 \%$ higher at $30^{\circ} \mathrm{C}$, the three different strains of $L$.

285 fermentum are favoured by higher temperatures, but with considerable differences among them.

286 Strain F1 had its growth rate leaping from 0.03 to 0.21 , a 5 fold increase in the growth rate.

287 Strain F2 shows a growth rate $76 \%$ higher at $37^{\circ} \mathrm{C}$, and Strain F3 a growth rate $170 \%$ higher

288 (Figure 4). These results suggest that the strain level dynamics of microbial populations is 
290 functionalities of microbiomes.

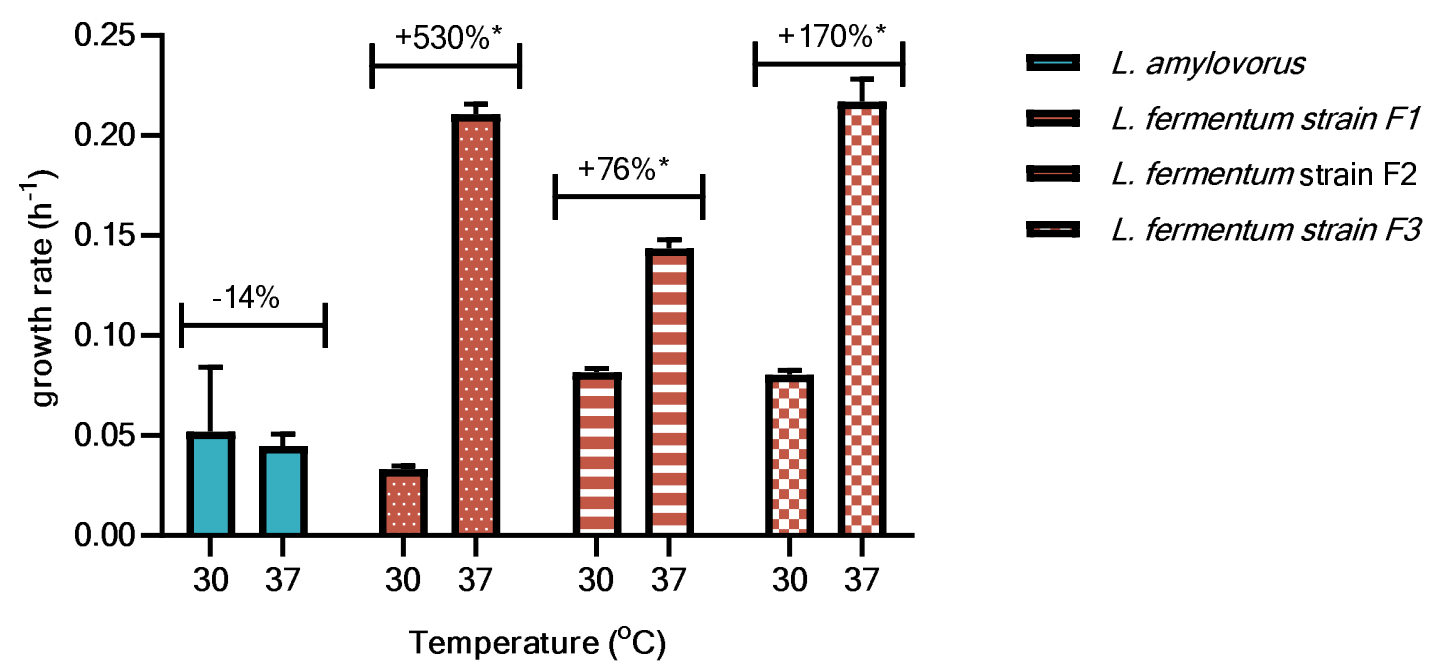

292 Figure 4: The influence of temperature in the growth rate $\left(h^{-1}\right)$ of different industrial

293 isolates. Higher fermentation temperatures favours L. fermentum in detriment of L. amylovorus,

294 which has its growth rate reduced in $14 \%$ (from $0.052 \pm 0.026$ to $0.045 \pm 0.005$ ). Within $L$.

295 fermentum, different strains display diverse responses to the increase of temperature. Strain F1

296 shows an average increase in its growth rate of $530 \%$ (from $0.33 \pm 0.001$ to $0.211 \pm 0.004$ ). Strain

297 F2 grows $76 \%$ faster (from $0.082 \pm 0.002$ to $0.143 \pm 0.004$ ), and strain $\mathrm{F} 3,170 \%$ (from $0.08 \pm 0.002$

298 to $0.217 \pm 0.009) . * \mathrm{p}<0.05$.

301 The comparison of the pangenomes of the 3 L. fermentum strain clusters revealed the presence of

302 functions and pathways not found in the other strains, which may be associated with their impact

303 and prevalence on the industrial process. (Methods, Supplementary Table 7). Strain clusters 1

304 and 3 (the most detrimental ones) present several unique genes which seem to be correlated to an 
305 adaptation for living and growing in the fermentation environment. Furthermore, strain cluster 1

306 contains arginine biosynthesis genes (KO1438), an amino acid that L. fermentum is otherwise

307 known to be auxotrophic for ${ }^{34}$. Ameliorating this auxotrophy could improve the competitive

308 fitness of this strain in an amino acid depleted environment ${ }^{35}$.

309

310 Loss of performance in fermentation is related to the competition between bacteria and

311 yeast, and the metabolite profile of $L$. fermentum strains

312 To elucidate the impact of strain variation on the ethanol fermentation yield by S. cerevisiae, we

313 performed static batch cultivations, using the model yeast strain PE-2 and L. fermentum strains

314 isolated from the samples used in our microbiome analyses (Figure 5A). Here, we conducted

315 pairwise fermentations that simulated the conditions of a typical industrial setup by applying a

316 yeast-to-bacteria ratio of $100: 1^{5}$, and using a chemically-semi defined synthetic medium that

317 resembles the sugarcane molasses-based broth ${ }^{36,37}$.

319 The yield obtained from the standalone fermentation by yeast PE-2 served as a control. To

320 further contextualise our findings, we repeated these experiments on the 6 most abundant

321 bacterial species (Figure 1B). Altogether, the species we analysed account for almost $80 \%$ of

322 known species in the contaminant microbiome (Supplementary Table 4).

324 Our results demonstrate that the presence of L. fermentum, compared to other abundant bacterial

325 species, can have a negative impact on fermentation performance ${ }^{20}$, with one strain decreasing

326 ethanol yield by $4.63 \pm 1.35 \%$ (Figure 5A). This contrasts with L. amylovorus, P. claussenii or L.

327 buchneri, which showed positive effects on ethanol yield (multiple t-test, $\mathrm{p}<0.05$ ). Both $L$. 
amylovorus and $P$. claussenii have a homofermentative metabolism, which has been shown to be

329 less detrimental to $S$. cerevisae in this fermentation setup ${ }^{6}$. Notably, L. buchneri strains have

330 previously been shown to produce considerable amounts of ethanol and lactate from glucose . $^{38}$

331 This ethanol production might contribute to greater ethanol titres and yields, despite competing

332 with yeast during the fermentation process. These observations are reinforced by our

333 metagenomics analyses, where the presence of these species correlated poorly with acidity,

334 which was strongly associated with lower ethanol yields (Figure 2B).

336 Interestingly, we provide experimental evidence that not all L. fermentum strains are detrimental,

337 as in the case of strain F2 $(91.38 \pm 3.04 \%)$, which was comparable to control $(89.23 \pm 0.23 \%$;

338 multiple t-test, $\mathrm{p}<0.05)$. When comparing the metabolite profile of supernatants from

339 monocultures of the L. fermentum isolate strains, we observe that there is a striking difference

340 between the somewhat neutral strain F1 and the beneficial strain F2 with the detrimental strain

341 F3. Not only its organic acid production titre is twice as the one observed for strains F1 and F2, it

342 also does not produce ethanol, which will have a direct impact on final ethanol titre and yield of

343 co-cultures with S. cerevisae (Figure 5B). These findings are in accordance to literature

344 observations, which suggest that the ratio between different organic acids is more important than

345 the high titres of specific organic acids for the inhibition of $S$. cerevisae ${ }^{27,39}$. This data

346 demonstrates the importance to monitor not just the total acidity, but also the composition of the

347 organic acid pool, in order to adapt the response to microbial contamination accordingly to this

348 particular data. 


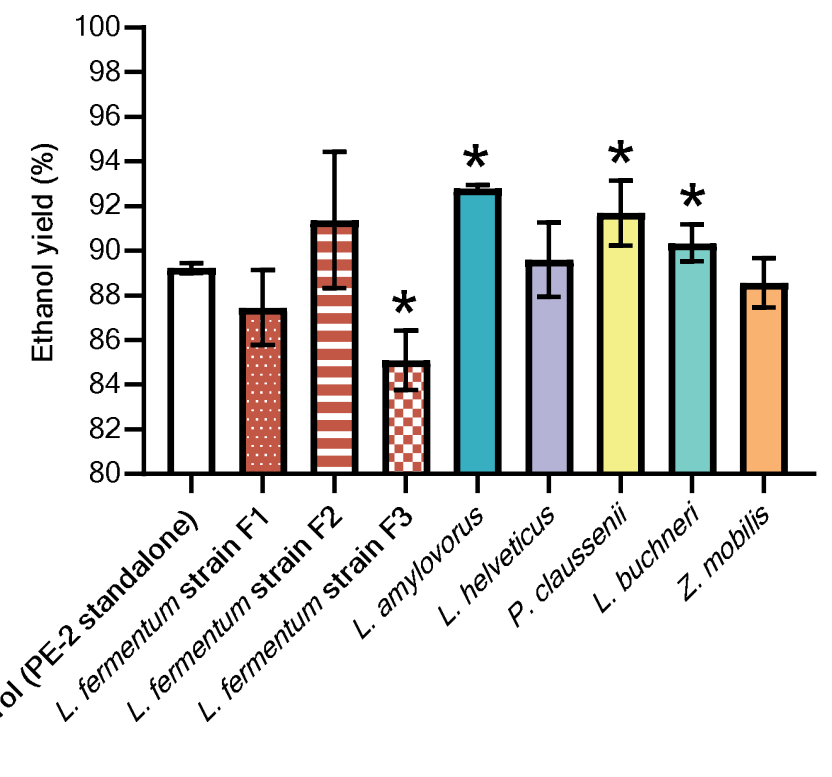

B

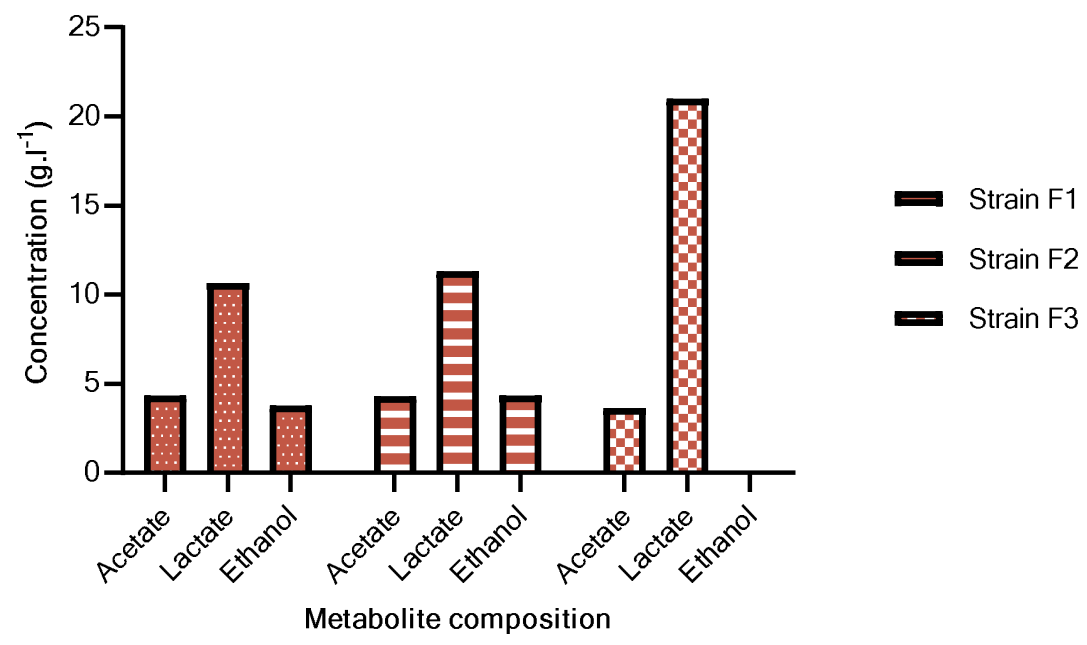

351 Figure 5: Specific L. fermentum strains cause reduction in bioethanol fermentation yield. A:

352 Ethanol yield of control fermentations (PE-2 yeast strain standalone) is compared against

353 pairwise fermentations with yeast strain PE-2 and 3 L. fermentum industrial isolates (filled bars);

354 and with each of the 5 most common contaminant bacteria found in sugarcane ethanol

355 fermentations. Of these species, which account for $80 \%$ of the contaminant microbial

356 community, only L. fermentum strains F1 and F3 reduce ethanol yield, albeit only F3 being 
statistically significant (by $1.98 \pm 1.68 \%$ and $4.63 \pm 1.35 \%$ respectively; multiple t-test). ${ }^{*} \mathrm{p}<0.05$.

358 B: The metabolite profile of the different L. fermentum strain isolates supernatants. Strains F1

359 and F2 show a similar metabolite production. On the other hand, strain F3 - the most detrimental

360 one - presents a remarkably different metabolite production, with twice as much lactate (up to 21

$361 \mathrm{~g} \mathrm{x} \mathrm{l}^{-1}$ ) and no ethanol produced, when compared to the other strains. This suggests that the mode

362 of inhibition of lactobacilli is directly correlated with their organic acid production profile ${ }^{6}$.

364 In the detrimental strain F3 genome we identified a unique gene cluster that is involved in 365 glycerol catabolism (KO2440, KO6120, KO6121 and KO6122). Glycerol is the second most 366 abundant metabolite produced by S. cerevisiae in alcoholic fermentations ${ }^{33}$. The ability to use 367 glycerol as an electron acceptor, as it is done by $L$. reuteri ${ }^{40}$, could likely provide a competitive 368 advantage for these strains when growing in the presence of yeast, allowing them to exploit this 369 exclusive niche created by yeast metabolism. Moreover, more of the carbon source could be 370 deviated towards biomass production instead of energetic metabolism, since this strain would not 371 require to reduce acetaldehyde into ethanol to rebalance the $\mathrm{NADH} / \mathrm{NAD}^{+}$pool, as it is 372 commonly done in lactobacilli. The metabolite profile data indeed suggests that such strain lacks 373 ethanol production under fermentative conditions and provides a mechanism for its particular 374 detrimental effects on overall ethanol yields. In order to identify functions or pathways in our $L$. 375 fermentum strains that may have contributed to their distinct fermentation behaviour, we 376 sequenced their genomes and conducted a comparative analysis (Methods, Supplementary

377 Table 7).The genetic diversity found between these strain clusters could allow, in theory, for the 378 development of modern diagnostic tools (e.g. qPCR analysis) which could predict the 379 performance of future fermentation batches, improving the process control of this industry. 


\section{Discussion}

382 Taken together, we have demonstrated that strain-level variation affects the output of a well-

383 controlled industrial bioethanol fermentation process. This highlights the importance of using

384 high-resolution, cross-sectional analysis of the contaminant microbiome in combination with

385 relevant industrial indicators. We observe the interplay between L. fermentum and L. amylovorus

386 that suggests competition between the two species in 2 ethanol producing mills. We also

387 demonstrate the utility of the industrial sugarcane ethanol fermentation as a model system to

388 study the dynamics and ecological interactions of microbial community, due to its highly

389 compartmentalized setup, which enables the impact of perturbations (such as strain-level

390 alterations) to be easily quantified.

391

392 Along these lines, we also introduce a novel metric by which to assess and compare the

393 performance of an industrial bioethanol fermentation batch. Using this metric, we associated

394 genetic variants of L. fermentum with fermentation efficiency, and identified the mechanism

395 underlying the prevalence of detrimental strains as being the temperature of fermentations.

396 Higher fermentation temperatures, above $32^{\circ} \mathrm{C}$, privilege specific L. fermentum strains, which

397 become the dominant strains in the population, and are considerably more detrimental to yeast

398 fermentation, due to their different metabolic profile. Selective removal of the identified strain

399 clusters could potentially improve ethanol yield by almost 5\%, translating to estimated economic

400 gains of 690 million $\mathrm{USD}^{41}$ and more than 2 million tons per year of $\mathrm{CO}_{2}$ emissions for the

401 Brazilian bioethanol industry ${ }^{42}$. 


\section{Acknowledgements}

404 The authors would like to acknowledge Marcos Vinícius, Thais Granço and Rafael Alves on

405 their support on acquiring industrial samples and metadata. The authors would like to

406 acknowledge Prof Dr Adriano Azzoni and Prof Dr René Schneider on providing access to

407 laboratory equipment for processing industrial samples. The authors would like to acknowledge

408 the support of Georges Neto on the processing of samples from the first sampling timepoint 409 batch.

410 The research was supported by funding from The Novo Nordisk Foundation under NFF grant

411 number: NNF10CC1016517. SSL acknowledges support from EMBO (ALTF 137-2018). TOB

412 acknowledges funding from FAPESP, with grant numbers: 2015/50684-9 and 2018/17172-2.

\section{Author contributions}

415 FSOL, MAM, KK, SSL, TOB, GP and MOAS designed the study. FSOL, TOB and BLVC 416 collected and processed industrial samples and metadata. FSOL prepared the metagenomics 417 samples for sequencing. MAM, KK and SSL performed bioinformatics analyses. MAM, KK, 418 SSL, FSOL, GP and MOAS critically analysed bioinformatics results. FSOL isolated industrial 419 strains. FSOL designed and performed laboratory scale fermentations. FSOL and MOAS 420 critically analysed the laboratory scale fermentation results. FSOL, MAM, KK, SSL and MOAS 421 wrote the manuscript. All authors have provided inputs for the manuscript.

\section{Competing interests}

424 The authors declare they have no competing interests. 


\section{Methods}

\section{Chemicals}

428 Unless stated otherwise, all chemicals and reagents used were purchased from Sigma-Aldrich 429 (St. Louis, MO, USA).

\section{$431 \quad$ Sampling strategy}

432 We sampled two independent ethanol mills (named Mill A and Mill B) in the production season

433 of 2017. Both mills are located in the State of São Paulo, Brazil - in a region with the prevalence

434 of the humid subtropical climate $(C f a)$ with an annual precipitation of around $2000 \mathrm{~mm}$, and with

435 a sea-level altitude of $c a$. 600m. The mills were completely independent from each other, with a

436 distance greater than $300 \mathrm{~km}$ apart, and have raw material sourced from different producers and

437 sugarcane fields. Both mills operated via fed-batch fermentations (Melle-Boinot setup), and had

438 a similar ethanol production capacity with a daily output of ca. $400 \mathrm{~m}^{3}$ of ethanol. Mill A was

439 sampled in the dates: 26/05/2017; 26/10/2017 and 17/11/2017. Mill B was sampled in the dates:

$440 \quad 02 / 06 / 2017 ; 29 / 10 / 2017$ and 03/11/2017. The following steps of the ethanol production process

441 were sampled: (1) Fermentation broth (Feeding line with fresh fermentation media); (2) start; (3)

442 middle; (4) end of fermentation; (5) yeast cream after separation of the wine, which is sent to

443 distillation centrifugation); and (6) biomass after acid wash treatment (sulphuric acid pH 2.5 for

444 1hour). The phases of the fermentation were defined according to the feeding regimen of each

445 mill: the beginning was set as the beginning to the middle of the feeding; the middle was defined

446 from the middle to the end of the feeding, and the end was defined as the final hours of

447 fermentation, after the feeding had ceased. Due to the fact that different vessels are fed 448 sequentially, we were able to sample different vats at different stages of fermentation, during the 
same sampling. Samples were collected directly from the production process and diluted $1 \mathrm{x}$ in a

450 sterile Phosphate Buffered Saline (PBS) solution with glycerol (50\%). The samples were readily

451 frozen in dry ice, until final storage in ultrafreezer $\left(-80^{\circ} \mathrm{C}\right)$. Each mill had several vessels

452 operating in the same fermentation step, which allowed for process replicates. Samples were

453 taken in duplicates.

\section{Industrial metadata}

456 The industrial metadata was provided by the operational staff from each mill, and consisted on

457 key process control parameters collected and registered by industrial staff, related to the ethanol

458 fermentation. Those parameters were: Ethanol yield daily average); ethanol yield (weekly

459 average); acidity from wine ( $g_{\text {acetic }}$ acid equivalent. $1^{-1}$, where $g_{\text {acetic }}$ acid equivalent is related to

460 the amount, in g. $\mathrm{l}^{-1}$, of acetic acid equivalent obtained via titration); yeast cell counts in the

461 fermentation (CFU); bacteria cell counts in the fermentation (CFU); yeast viability (\% of the

462 population); yeast budding rate (\% of the population); vessel current volume (in $\mathrm{m}^{3}$ ); vessel

463 operational status (idle, feeding, running or finished fermentation) and vessel temperature (in $\left.464{ }^{\circ} \mathrm{C}\right)$.

465 For correlation analyses, the data was converted into monthly averages.

467 DNA extraction and sequencing of isolates and metagenomes

468 All DNA extractions were performed using the DNeasy Powerlyzer Powersoil Kit (QIAGEN, 469 Hilden, Germany), according to manufacturer's instructions. Pure lactobacilli isolates had their

470 DNA extracted using MasterPure $^{\mathrm{TM}}$ Gram Positive Purification Kit (LGC Biosearch

471 Technologies, Hoddesdon, UK). All DNA extraction quantifications were performed with Qubit 
472 Fluorometer (Thermo Fischer Scientific, Waltham, MS, USA). Due to low biomass content,

473 DNA extraction was not possible for broth sample (Process Step 1) from Mill A at the $3^{\text {rd }}$

474 sampling timepoint. Shotgun metagenomics and isolates genome sequencing was performed on

475 the NextSeq 500 using NextSeq High Output v2 Kit (300 Cycles) (Illumina, San Diego, CA,

476 USA) by the Sequencing Core Facility at The Novo Nordisk Foundation Center for

477 Biosustainability (Technical University of Denmark, Kongens Lyngby, Denmark). The library

478 preparation was performed using the KAPA HyperPlus Library Prep Kit (Roche, Basel,

479 Switzerland), and the indexing kit used was the Dual Indexed PentAdapters, Illumina compatible

480 (PentaBase, Odense, Denmark). Quantity and quality control were performed using Qubit

481 dsDNA HS Assay Kit (Invitrogen, Carlsbad, CA, USA) and DNF-473 Standard Sensitivity NGS

482 Fragment Analysis Kit (1 bp - 6000 bp; Agilent, Santa Clara, CA, USA). Average library length

483 was $341 \mathrm{bp}$. The sequencing reads length were 150 base pair paired-end (2x150 bp). The index

484 (i7 and i5) reads were $8 \mathrm{bp}$, dual indexed and flow cell loading was $1.3 \mathrm{pM}$. The sequencing

485 chemistry used was 2-channel sequencing-by-synthesis (SBS) technology, and Phix control V3

486 (Illumina San Diego, CA, USA) was added (2.5\%).

\section{Processing of genomic and metagenomic data}

489 Raw reads (from both metagenomic and isolate sequencing) underwent quality trimming, i.e.

490 filter out adapter and universal primer sequences, as well as low quality bases (< Q20), reads

491 shorter than 75 bp and duplicated reads (Supplementary Table 2), as previously described ${ }^{43}$.

492 All 481 S. cerevisiae genomes from NCBI genome database (August 2018) were downloaded.

493 Reads were aligned to concatenated genomes using the BWA mem model with default

494 parameters $^{43}$. Reads over $95 \%$ identity were considered to belong to S. cerevisiae (SC reads) and 
495 not used in our analyses (Supplementary Table 2). Kraken ${ }^{44}$ was selected for taxonomic read

496 assignment of non-SC reads as it has been shown to perform well in benchmarking studies ${ }^{45,46}$,

497 especially for medium-low complexity microbiome communties ${ }^{47}$ such as the ones in industrial

498 fermenters. Specifically, Kraken v. 0.10.5-beta was applied on non-yeast reads with default

499 settings against the minikraken 2017.10.18 8GB database. Bracken ${ }^{48}$ v. 2.0 was used for accurate

500 species abundance estimation with parameters -r (read length) 150 and -1 (level) S (species).

501

502 Analyses of metagenomics data

503 Rarefaction of read counts and subsequent analyses were done using $\mathrm{R}$ package vegan ${ }^{49}$. We

504 considered the 10 most prevalent contaminant species as those with the highest median relative

505 abundance across all samples. Microbial community compositions were compared using Bray-

506 Curtis distance on species relative abundance and Permutational Multivariate Analysis of

507 Variance (PERMANOVA) with 999 permutations and the Bray-Curtis method was applied by

508 providing Mill/Process step/Date as function. Pairwise Spearman's correlation coefficient was

509 calculated for pairs of metadata variables, and between metadata variables and taxon

510 abundances. False discovery rate (FDR) was calculated using Benjamini-Hochberg (BH)

511 method, with FDR $<0.05$ used as cut-off.

\section{Strain level profiling}

514 To profile the strains within a bacterial species, we used a published pipeline ${ }^{43,50}$ with the

515 following modifications: (1) all contigs from one NCBI genome were concatenated as a

516 consecutive sequence with spacers ( $\mathrm{N}$ of 100bp); (2) genome assemblies from the NCBI

517 database were automatically downloaded and renamed; (3) the new NCBI accession ID system 
518 in place of the old sequence ID and taxonomic ID system, which allowed more assemblies to be

519 included. For each species, genomes were downloaded from the NCBI Genome database

520 (August 2018) to construct a SNV and pan-genome database. In cases where more than 200

521 genome assemblies existed, only complete assemblies and chromosome-level assemblies were

522 used. The SNV-based core-genome strain profiling was used for downstream analyses. Strains

523 with a maximum relative abundance less than $5 \%$ in at least one sample, or present in less than

$52420 \%$ samples were discarded.

525 For each species, Spearman's correlation coefficient was calculated among different strains'

526 relative abundance of all metagenomic samples. False discovery rate (FDR) was calculated using

527 Benjamini-Hochberg $(\mathrm{BH})$ method. FDR $<0.05$ was used as the significant level cut-off. Strain

528 abundance dissimilarity among different samples was calculated by Euclidean distances and

529 hierarchical clustering was performed. As a result, all strains were clustered into two to six strain

530 clusters for each species, where no significant negative correlation could be captured in each

531 strain cluster (see Supplementary Figure $\mathbf{S 3}$ as an example from L. fermentum).

\section{Industrial performance calculation}

534 The industrial performance calculation was obtained by the product of the multiplication of the

535 parameters directed correlated with process performance (i.e. ethanol yield and yeast viability),

536 divided by the product of the multiplication of the parameters inversely correlated with process

537 performance (i.e. bacterial cell counts and acidity titre):

$$
\text { Industrial performance }=\frac{\left(\text { Eth }_{\text {yield }} \mathrm{x} \text { Yeast }_{\text {viab }}\right)}{\left(\text { Bac }_{\text {counts }} \times \text { Acid }_{\text {titre }}\right)}
$$

538 Equation 1: Proposed equation for obtaining a general industrial performance score. The

539 score is obtained by multiplying ethanol yield $\left(\right.$ Eth $\left._{\text {yield }}\right)$ and yeast viability (Yeast $\left.{ }_{\text {viab }}\right)$ values, and 
540 dividing its product by the product obtained from the multiplication of bacterial cell counts

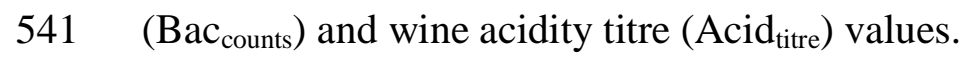

\section{Strains used in laboratory experiments}

544 Saccharomyces cerevisiae strain PE-2 was kindly provided by Prof. Thiago Olitta Basso. Strains

545 of Lactobacillus amylovorus and Lactobacillus fermentum were isolated from stored industrial 546 samples. Strains of Pediococcus claussenii, Lactobacillus helveticus, Lactobacillus buchneri and

547 Zymomonas mobilis were purchased from ATCC (Manassas, VA, USA).

\section{Isolation and maintenance of industrial strains}

550 For strain isolation, a previously introduced protocol was used ${ }^{13}$. Briefly, industrial samples were 551 serially diluted in sterile PBS and plated in Man Rogosa Sharpe (MRS) Agar media, containing 552 cycloheximide $\left(0.1 \% \mathrm{v} \cdot \mathrm{v}^{-1}\right)$ in order to inhibit yeast growth. Plates were incubated at either $30^{\circ} \mathrm{C}$ 553 or $37^{\circ} \mathrm{C}$ statically. A loopful of an isolated colony was grown in liquid MRS in the same 554 conditions, and stored at $-80^{\circ} \mathrm{C}$ (see section "DNA extraction and analysis of bacterial isolates"). 555 Yeast strains were cultured in Yeast Potato Dextrose (YPD) media, at $30^{\circ} \mathrm{C}$. Lactobacilli were 556 cultured in MRS media, either at $30^{\circ} \mathrm{C}$ or $37^{\circ} \mathrm{C}$, and Zymomonas mobilis was cultured at Trypsin 557 Soy Broth (TSB) media, at $30^{\circ} \mathrm{C}$. All cultivations were performed statically, in $\mathrm{ca}$. $5 \mathrm{ml}$ volume.

\section{DNA extraction and analysis of bacterial isolates}

560 Pure isolates were grown overnight in adequate media and conditions, as mentioned in the 561 previous section. After growth, cells were pelleted via centrifugation (> 10,000g for 4 min.) and 
562 their genomic DNA was extracted using the MasterPure ${ }^{\mathrm{TM}}$ Gram Positive DNA Purification Kit

563 (Lucigen Corporation, Middleton, WI), according to manual's instruction.

564

\section{Bacterial isolate assembly}

566 A de Bruijn graph-based assembler, SPAdes $3.12^{51}$, was used for the genome assembly of 567 bacterial isolates, using the following parameters: “-m 300 -k 33,55,77,99,127”. To complement 568 the de novo assembly, reference-assisted genome assembly was performed with idba_hybrid (v $5691.1 .1)^{52}$ with the following parameters “--pre_correction --mink 120 --maxk 180 --step 10 -570 min_contig 300 --reference [the reference genome downloaded from NCBI for each species]".

571 Two modifications were made in the source code before compiling IDBA_UD: in file 572 src/basic/kmer.h constant kNumUint64 was changed from 4 to 8 to allow maximum kmer length 573 beyond 124; in file src/sequence/short_sequence.h constant kMaxShortSequence was set to 512 574 to support longer read length. Final assembly results were summarized in Supplementary Table 575 4, Methods.

\section{Genome-based functional analysis}

578 ORFs were predicted on assembled strain genomes using MetaGeneMark v.3.26 ${ }^{53}$. Predicted 579 proteins were annotated using eggNOG mapper ${ }^{54}$ with the following settings: mapping mode: 580 DIAMOND, automatic taxonomic scope, orthologs: restrict to one-to-one (prioritize precision),

581 GO evidence: use experimental-only terms (prioritize quality). From the resulting file, KEGG 582 ortholog ids were extracted by significant matches (e-value $<10^{-5}$ ) and those that were unique

583 for each strain cluster were characterized in terms of KEGG pathway/module membership 584 (KEGG Mapper Pathway Reconstruct) ${ }^{55}$. 


\section{Fermentation experiments}

587 Fermentations were performed in 96 deep-well plates, with either pairwise cultivations

588 (yeast:bacteria at a 100:1 ratio) ${ }^{8}$, or standalone yeast or bacteria cultivations. The media used is a

589 semi-synthetic media, able to simulate sugarcane molasses based media $(\mathrm{SM})^{37}$. Briefly, all

590 strains were cultured in their optimal media and conditions (see "Strains" and "Isolation of

591 industrial strains and maintenance" sections), for up to $48 \mathrm{~h}$. After that, the biomass was

592 calculated via optical density (OD; $600 \mathrm{~nm}$ wavelength). All cells were pelleted via

593 centrifugation $\left(3400 \times \mathrm{g}, 4^{\circ} \mathrm{C}, 15 \mathrm{~min}\right)$ and washed twice with sterile PBS. Subsequently, cells

594 were diluted in SM diluted in sterile Milli-Q H2O (10x, final sugar concentration of $\left.18 \mathrm{~g} .1^{-1}\right)$ for

595 an OD value of 1.0. Strains were later diluted in fresh SM media in specific wells in the 96 deep-

596 well plate to a final OD value of 0.1 .

597 The lactobacilli growth rate analysis was performed at 30 and $37^{\circ} \mathrm{C}$, under agitation (double

598 orbital, fast mode) in Synergy H1 plate readers (Biotek Instruments, Inc. Winooski, VT, USA).

599 OD was measured every 30 minutes for $24 \mathrm{~h}$. The growth rate was later calculated using the R

600 package growthcurver ${ }^{56}$.

601

602 All the pairwise cultivations were performed statically, overnight, at $30^{\circ} \mathrm{C}$, in ca. $1 \mathrm{ml}$ volume.

603 The fermentations were performed in triplicate. The carbohydrate titre and composition (sucrose,

604 glucose and fructose) and fermentation metabolites (glycerol, ethanol, and acetic acid) were

605 determined by high-performance liquid chromatography (HPLC) (UltiMate 3000, Thermo-

606 Fischer Scientific, Waltham, Massachusetts, USA). The analites were separated using an Aminex

607 HPX-87H ion exclusion column (Bio-Rad, Hercules, California, USA) and were isocratically 
608 eluted at $30^{\circ} \mathrm{C}$, with a flow rate of $0.6 \mathrm{ml} \cdot \mathrm{min}^{-1}$, using a $5 \mathrm{mM}$ sulphuric acid solution as mobile

609 phase. The detection was performed refractrometrically.

610 Ethanol yield was calculated according using the following equation:

$$
\text { Ethanol yield }=\frac{\left(\mathrm{EtOH}_{\mathrm{obs}} \times 100\right)}{\mathrm{EtOH}_{\text {theor }}}
$$

611 Equation 2: Ethanol yield calculation. Where: $\mathrm{EtOH}_{\mathrm{obs}}=$ the observed ethanol titre on each

612 sample. $\mathrm{EtOH}_{\text {theor }}=$ the maximum theoretical ethanol titre for each sample. Obtained by

613 multiplying the sugar titre from the broth solution with the stoichiometric conversion factor for

614 ethanol production (i.e. 0.5111$)^{57}$.

615

616 Community composition was resolved via flow-cytometry (BD LSRFortessa ${ }^{\mathrm{TM}}$, BD Biosciences,

617 Franklin Lakes, New Jersey, USA). A sample from each well (10 $\mu$ l) was taken after the

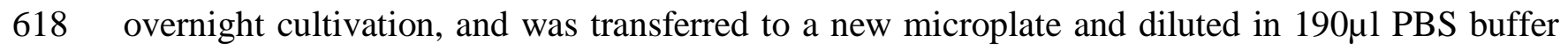

619 (pH 7.4). Yeast and bacteria populations were resolved via front and side scatter comparison

620 (SSC versus FSC). The statistical analyses were performed using the software GraphPad Prism

621 8. The difference on final ethanol yield was analysed by multiple t-tests (statistical significance

622 analysis with alpha value of 0.05$)$.

624 Lactobacilli supernatant metabolite profile was analysed via HPLC after 48h of growth, using the

625 aforementioned analytical method. A pre-inoculum of lactobacilli stored at $-80^{\circ} \mathrm{C}$ was grown in

626 MRS for 24h. After that the OD from these cultures was measured and fresh MRS media was

627 inoculated with a fixed OD of 0.1 and incubate statically at $37^{\circ} \mathrm{C}$. After growth, the cells were

628 separated via centrifugation and the supernatant was sent for further analysis. 


\section{Metagenomic co-assembly and functional annotation}

631 To overcome the imbalance between the sequencing yields of bacterial and fungal reads in

632 different samples, achieve higher completeness of pan-genome regions with low sequencing

633 coverage, and perform sample-wise gene presence and absence comparisons, co-assembly was

634 performed for the non-SC reads. Non-SC reads were concatenated separately from all sequenced

635 samples and the maximum $k$-mer depth was normalized to 100 fold by BBnorm

636 (https://sourceforge.net/projects/bbmap/) before co-assembly. IDBA_ud (v. 1.1.1) ${ }^{52}$ was used for

637 the assembly using the following parameters: “--min_contig 300 --mink 50 --maxk 124 --step 10

638 --pre_correction”. Co-assembly results are summarized in Supplementary Table 3, Methods.

639 For the non-SC assembly, MetaGeneMark v. 3.26 was adopted to predict the coding DNA

640 sequence (CDS) regions in the assembled metagenome contigs using the default parameters.

\section{References}

643 1. Silvério da Silva, S. \& Chandel, A. K. Biofuels in Brazil. (Springer, 2014).

644 doi:10.1007/978-3-319-05020-1

645 2. Wang, M., Han, J., Dunn, J. B., Cai, H. \& Elgowainy, A. Well-to-wheels energy use and 646 greenhouse gas emissions of ethanol from corn, sugarcane and cellulosic biomass for US

647 use. Environ. Res. Lett. 7, 13 (2012).

648 3. Wheals, A. E., Basso, L. C., Alves, D. M. G. \& Amorim, H. V. Fuel ethanol after 25

$649 \quad$ years. Trends Biotechnol. 17, 482-487 (1999).

650 4. Basso, L. C., Basso, T. O. \& Rocha, S. N. Ethanol Production in Brazil $\square$ : The Industrial 651 Process and Its Impact on Yeast Fermentation. Biofuel Prod. Recent Dev. Prospect. 1530, $652 \quad$ 85-100 (2011). 
653 5. Amorim, H. V. et al. Scientific challenges of bioethanol production in Brazil. Appl.

$654 \quad$ Microbiol. Biotechnol. 91, 1267-1275 (2011).

655 6. Basso, T. O. et al. Homo-and heterofermentative lactobacilli differently affect sugarcane-

656 based fuel ethanol fermentation. Antonie van Leewenhoek 105, 169-177 (2014).

657 7. Lopes, M. L. et al. Ethanol production in Brazil: a bridge between science and industry.

658 Brazilian J. Microbiol. 47, 64-76 (2016).

659 8. Ceccato-Antonini, S. R. Conventional and nonconventional strategies for controlling

660 bacterial contamination in fuel ethanol fermentations. World J. Microbiol. Biotechnol. 34,

$66180(2018)$.

662 9. Stubbendieck, R. M., Vargas-Bautista, C. \& Straight, P. D. Bacterial Communities:

$663 \quad$ Interactions to Scale. Front. Microbiol. 7, 1234 (2016).

664 10. Sinsabaugh, R. L., Manzoni, S., Moorhead, D. L. \& Richter, A. Carbon use efficiency of 665 microbial communities: stoichiometry, methodology and modelling. Ecol. Lett. 16, 930$666939(2013)$.

667 11. Ait Mueller, J. et al. Interspecies interactions are an integral determinant of microbial 668 community dynamics. Front. Microbiol. 6, 1-11 (2015).

669 12. Makanjuola, D. B., Tymon, A. \& Springham, D. G. Some effects of lactic acid bacteria on 670 laboratory-scale yeast fermentations. Enzyme Microb. Technol. 14, 350-357 (1992).

671 13. Lucena, B. T. L. et al. Diversity of lactic acid bacteria of the bioethanol process. $B M C$ $672 \quad$ Microbiol. 10, 298 (2010).

673 14. Bischoff, K. M., Liu, S., Leathers, T. D., Worthington, R. E. \& Rich, J. O. Modeling 674 bacterial contamination of fuel ethanol fermentation. Biotechnol. Bioeng. 103, 117-122 675 (2009). 
676 15. Skinner, K. A. \& Leathers, T. D. Bacterial contaminants of fuel ethanol production. J. Ind.

677 Microbiol. Biotechnol. 31, 401-408 (2004).

678 16. Beckner, M., Ivey, M. L. \& Phister, T. G. Microbial contamination of fuel ethanol

679 fermentations. Lett. Appl. Microbiol. 53, 387-394 (2011).

680 17. Bonatelli, M. L., Quecine, M. C., Silva, M. S. \& Labate, C. A. Characterization of the

681 contaminant bacterial communities in sugarcane first-generation industrial ethanol

682 production. FEMS Microbiol. Lett. 364, 1-8 (2017).

683 18. Costa, O. Y. A. et al. Microbial diversity in sugarcane ethanol production in a Brazilian

684 distillery using a culture-independent method. J. Ind. Microbiol. Biotechnol. 42, 73-84 (2015).

686 19. Narendranath, N. V., Hynes, S. H., Thomas, K. C. \& Ingledew, W. M. Effects of lactobacilli on yeast-catalyzed ethanol fermentations. Appl. Environ. Microbiol. 63, 41584163 (1997).

689 20. Rich, J. O., Leathers, T. D., Bischoff, Kenneth, M., Anderson, A. M. \& Nunnally, M. S. Biofilm formation and ethanol inhibition by bacterial contaminants of biofuel

691 fermentation. Bioresour. Technol. 196, 347-354 (2015).

692 21. Yang, C. et al. Strain-level differences in gut microbiome composition determine fecal

$693 \quad$ IgA levels and are modifiable by gut microbiota manipulation. bioRxiv 544015 (2019).

694 doi: $10.1101 / 544015$

695 22. Koch, C., Müller, S., Harms, H. \& Harnisch, F. Microbiomes in bioenergy production:

696 From analysis to management. Curr. Opin. Biotechnol. 27, 65-72 (2014).

697 23. Leathers, T. D. et al. Inhibitors of biofilm formation by biofuel fermentation

698 contaminants. Bioresour. Technol. 169, 45-51 (2014). 
699 24. Li, Q., Heist, E. P. \& Moe, L. A. Bacterial Community Structure and Dynamics During

700 Corn-Based Bioethanol Fermentation. Microb. Ecol. 71, 409-421 (2016).

701 25. Andrietta, S. R. R., Andrietta, M. G. S. \& Bicudo, M. H. P. Comparação do rendimento

702 fermentativo utilizando diferentes metodologias de cálculo para avaliação do desempenho

703 de um processo industrial. STAB 30, 41-49 (2012).

704 26. Bhalla, A., Bischoff, K. M. \& Sani, R. K. Highly thermostable xylanase production from a 705 thermophilic Geobacillus sp. strain WSUCF1 utilizing lignocellulosic biomass. Front.

706 Bioeng. Biotechnol. 3, 1-8 (2015).

707 27. Piper, P., Calderon, C. O., Hatzixanthis, K. \& Mollapour, M. Weak acid adaptation $\square$ : the

708 stress response that confers yeasts with resistance to organic acid food preservatives.

$709 \quad$ Microbiology 147, 2635-2642 (2001).

710 28. Liu, M. et al. Bacteriophage application restores ethanol fermentation characteristics

711 disrupted by Lactobacillus fermentum. Biotechnol. Biofuels 8, 132 (2015).

712 29. Bassi, A. P. G., Meneguello, L., Paraluppi, A. L., Sanches, B. C. P. \& Ceccato-Antonini,

713 S. R. Interaction of Saccharomyces cerevisiae-Lactobacillus fermentum-Dekkera

714 bruxellensis and feedstock on fuel ethanol fermentation. Antonie Van Leeuwenhoek 111,

715 1661-1672 (2018).

716 30. Reis, V. R. et al. Effects of feedstock and co-culture of Lactobacillus fermentum and wild 717 Saccharomyces cerevisiae strain during fuel ethanol fermentation by the industrial yeast

718 strain PE-2. AMB Express 8, 1-11 (2018).

719 31. Chang, I., Kim, B.-H., Shin, P.-K. \& Lee, W.-K. Bacterial contamination and its effects on 720 ethanol fermentation. J. Microbiol. Biotechnol. 5, 309-314 (1995).

721 32. Carvalho-Netto, O. V et al. Saccharomyces cerevisiae transcriptional reprograming due to 
bacterial contamination during industrial scale bioethanol production. Microb. Cell Fact.

$723 \mathbf{1 4}, 13(2015)$.

724 33. Eli Della-Bianca, B., Olitta Basso, T., Ugarte Stambuk, B., Carlos Basso, L. \& Karoly

725 Gombert, A. What do we know about the yeast strains from the Brazilian fuel ethanol

726 industry? Appl. Microbiol. Biotechnol. 97, 979-991 (2013).

727 34. Kuratsu, M., Hamano, Y. \& Dairi, T. Analysis of the Lactobacillus metabolic pathway.

728 Appl. Environ. Microbiol. 76, 7299-301 (2010).

729 35. Walford, S. Composition of cane juice. Proc. South African Sugar Technol. Assoc. 70, 265-266 (1996).

731 36. Basso, L. C., De Amorim, H. V., De Oliveira, A. J. \& Lopes, M. L. Yeast selection for 732 fuel ethanol production in Brazil. FEMS Yeast Res. 8, 1155-1163 (2008).

733 37. Lino, F. S. de O., Basso, T. O. \& Sommer, M. O. A. A synthetic medium to simulate sugarcane molasses. Biotechnol. Biofuels 11, 221 (2018).

735 38. Liu, S., Skinner-Nemec, K. A. \& Leathers, T. D. Lactobacillus buchneri strain NRRL B73630929 converts a concentrated mixture of xylose and glucose into ethanol and other 737 products. J. Ind. Microbiol. Biotechnol. 35, 75-81 (2008).

738 39. Nicolaou, S. A., Gaida, S. M. \& Papoutsakis, E. T. A comparative view of metabolite and substrate stress and tolerance in microbial bioprocessing: From biofuels and chemicals, to

741 40. Stolz, P., Vogel, R. F. \& Hammes, W. P. Utilization of electron acceptors by lactobacilli

742 isolated from sourdough. Z. Lebensm. Unters. Forsch. 201, 402-410 (1995).

743 41. Portal Unica. Available at: http://www.unica.com.br/. (Accessed: 6th February 2019)

744 42. Börjesson, P. Good or bad bioethanol from a greenhouse gas perspective - What 
determines this? Appl. Energy 86, 589-594 (2009).

746 43. Kang, K. et al. The Environmental Exposures and Inner- and Intercity Traffic Flows of the Metro System May Contribute to the Skin Microbiome and Resistome. Cell Rep. 24, 1190-1202.e5 (2018).

749 44. Wood, D. E. \& Salzberg, S. L. Kraken: ultrafast metagenomic sequence classification using exact alignments. Genome Biol. 15, R46 (2014).

751 45. Escobar-Zepeda, A. et al. Analysis of sequencing strategies and tools for taxonomic annotation: Defining standards for progressive metagenomics. Sci. Rep. 8, 12034 (2018).

753 46. Gardner, P. P. et al. Identifying accurate metagenome and amplicon software via a metaanalysis of sequence to taxonomy benchmarking studies. PeerJ 7, e6160 (2019).

755 47. Sczyrba, A. et al. Critical Assessment of Metagenome Interpretation-a benchmark of 756 metagenomics software. Nat. Methods 14, 1063-1071 (2017).

757 48. Lu, J., Breitwieser, F. P., Thielen, P. \& Salzberg, S. L. Bracken: estimating species 758 abundance in metagenomics data. PeerJ Comput. Sci. 3, e104 (2017).

759 49. Dixon, P. VEGAN, a package of R functions for community ecology. J. Veg. Sci. 14, $760 \quad 927-930(2003)$.

761 50. Oh, J. et al. Biogeography and individuality shape function in the human skin 762 metagenome. Nature 514, 59-64 (2014).

763 51. Bankevich, A. et al. SPAdes: a new genome assembly algorithm and its applications to 764 single-cell sequencing. J. Comput. Biol. 19, 455-77 (2012).

765 52. Peng, Y., Leung, H. C. M., Yiu, S. M. \& Chin, F. Y. L. IDBA-UD: a de novo assembler 766 for single-cell and metagenomic sequencing data with highly uneven depth.

767 Bioinformatics 28, 1420-1428 (2012). 
768 53. Zhu, W., Lomsadze, A. \& Borodovsky, M. Ab initio gene identification in metagenomic

$769 \quad$ sequences. Nucleic Acids Res. 38, e132 (2010).

770 54. Huerta-Cepas, J. et al. Fast Genome-Wide Functional Annotation through Orthology

771 Assignment by eggNOG-Mapper. Mol. Biol. Evol. 34, 2115-2122 (2017).

772 55. Kanehisa, M., Furumichi, M., Tanabe, M., Sato, Y. \& Morishima, K. KEGG: New

773 perspectives on genomes, pathways, diseases and drugs. Nucleic Acids Res. 45, D353-

774 D361 (2017).

775 56. Sprouffske, K. \& Wagner, A. Growthcurver: An R package for obtaining interpretable

776 metrics from microbial growth curves. BMC Bioinformatics 17, 1-4 (2016).

777 57. Raghavendran, V., Basso, T. P., da Silva, J. B., Basso, L. C. \& Gombert, A. K. A simple

778 scaled down system to mimic the industrial production of first generation fuel ethanol in

779 Brazil. Antonie Van Leeuwenhoek 110, 971-983 (2017). 